\title{
Electrospun bundled carbon nanofibers for skin-inspired tactile sensing, proprioception and gesture tracking applications
}

\author{
Debarun Sengupta (iD) , Joshua Romano ${ }^{1}$ and Ajay Giri Prakash Kottapalli $\mathbb{D}^{1,2}$
}

In this work, we report a class of wearable, stitchable, and sensitive carbon nanofiber (CNF)-polydimethylsiloxane (PDMS) composite-based piezoresistive sensors realized by carbonizing electrospun polyacrylonitrile (PAN) nanofibers and subsequently embedding in PDMS elastomeric thin films. Electro-mechanical tactile sensing characterization of the resulting piezoresistive strain sensors revealed a linear response with an average force sensitivity of $\sim 1.82 \mathrm{kN}^{-1}$ for normal forces up to $20 \mathrm{~N}$. The real-time functionality of the CNF-PDMS composite sensors in wearable body sensor networks and advanced bionic skin applications was demonstrated through human motion and gesture monitoring experiments. A skin-inspired artificial soft sensor capable of demonstrating proprioceptive and tactile sensory perception utilizing CNF bundles has been shown. Furthermore, a 16-point pressure-sensitive flexible sensor array mimicking slow adapting low threshold mechanoreceptors of glabrous skin was demonstrated. Such devices in tandem with neuromorphic circuits can potentially recreate the sense of touch in robotic arms and restore somatosensory perception in amputees.

npj Flexible Electronics (2021)5:29; https://doi.org/10.1038/s41528-021-00126-8

\section{INTRODUCTION}

With the recent rapid progress in wearable electronic sensor technology real-time human motion monitoring, sensorized prosthetics, active patient biofeedback, and advanced bionic skins are becoming a reality. In recent years, flexible electronics and wearable human motion monitoring devices have emerged to be the most sought-after devices as they can potentially provide a host of valuable information regarding the health and well-being of an individual. For instance, gait monitoring in individuals suffering from neurological ailments such as Huntington's disease, multiple sclerosis, Parkinson's disease, and other associated neurological conditions can provide valuable information regarding the progression of the disease and hence, continuous monitoring of gait characteristics can facilitate early diagnosis, biofeedback and subsequently enable the healthcare providers to tailor patient-specific treatment plans ${ }^{1}$. Accurate monitoring of human locomotion and physiological parameters like breathing pattern, posture, involuntary muscle tremors, gait, joint and limb movements necessitates the availability of inexpensive, highly flexible, stretchable, lightweight, skin-mountable, and durable sensors. However, the limited flexibility and low resolution of the conventional semiconductor and metallic foil-based strain gauges restrict their applicability in tasks involving wide strain range like human joint movements. Also, conventional semiconductor and metallic strain gauges cannot meet the demands of wide-range strain sensing associated with human motion detection due to their limited detection range which is typically less than $5 \%{ }^{2-5}$. To circumvent the flexibility, low resolution and sensing range issues associated with the conventional strain gauges, various attempts have been made in the past to investigate suitable nanomaterialselastomer composites for developing flexible strain sensors customized for wearable devices. Flexible substrates utilizing polymer materials like ecoflex, polydimethylsiloxane (PDMS), polyurethane (PU), and polyimide (PI) have been used extensively due to their excellent responsiveness to torsion, compression, tension, and superior mechanical properties ${ }^{6-10}$. Various conductive nanomaterials like silver nanowires $(A g N W s)^{11,12}$, silicon nanoribbons $^{13}$, and various carbonaceous materials have been investigated in the past for developing flexible nanomaterialpolymer composite-based sensors. In particular, low-dimensional carbon-based materials like graphene, carbon nanotubes (CNTs), and carbon blacks (CBs) have been explored by researchers because of their superior conductivity, stiffness, and elastic properties ${ }^{6,9,14-21}$, Often the processes for synthesizing the nanomaterial fillers such as CNTs and graphene involve complicated processing steps such as chemical vapor deposition (CVD), mechanical and chemical exfoliation, graphite oxidation-reduction, arc discharge, and laser ablation ${ }^{9,22-26}$. Previously, Pang et al. reported a highly sensitive graphene porous network PDMS sensor fabricated by growing graphene inside the pores of nickel-PDMS foam having both pressure and strain sensing properties ${ }^{9}$. Zhang et al. proposed a novel method entailing a simple dip coating process and subsequent pre-compression treatment for fabricating cracked cellulose nanofibril/silver nanowire-coated PU sponges for piezoresistive sensing applications ${ }^{12}$. Liu et al. reported a $90 \%$ porous graphene-thermoplastic PU foams fabricated employing thermal induced phase separation technique for piezoresistive sensing applications ${ }^{16}$. Ryu et al. proposed a highly stretchable device fabricated by growing CNT fibers grown on ecoflex substrate $^{22}$. The reported device was capable of retaining a high sensitivity over a large strain range of $0-900 \%$. More recently MXene has been used in conjunction with polymers to develop MXene-polymer composites for both piezoresistive and capacitive sensing applications ${ }^{27-29}$. Ma et al. reported a highly flexible and sensitive MXene-based piezoresistive sensor demonstrating a high gauge factor of $180.1^{27}$. In addition to carbonaceous materials commonly used for piezoresistive tactile sensing, researchers have used piezoelectric materials and triboelectric effect for flow,

\footnotetext{
${ }^{1}$ Department of Advanced Production Engineering (APE), Engineering and Technology Institute Groningen (ENTEG), University of Groningen, Groningen 9747 AG, The Netherlands. ${ }^{2}$ MIT Sea Grant College Program, Massachusetts Institute of Technology (MIT), 77 Massachusetts Avenue, NW98-151, Cambridge, MA 02139, USA.

凶email: a.g.p.kottapalli@rug.nl
} 
impact, touch, and tactile sensing applications ${ }^{30-34}$. In most of the nanomaterial-polymer composites-based flexible sensors, the nanomaterial fillers are dispersed in suitable solvents and then deposited in the polymer substrate by patterning or dip-coating which often involves time-consuming and complicated processing steps which potentially throttle the commercialization prospects of such devices. In addition, the nature of the dip coating processes renders the sensor unstable as the active nanofillers tend to fall out under external repetitive loading leading to baseline resistance change over time. Furthermore, the high moduli $(\sim 1 \mathrm{TPa})$ of graphene and CNTs, render their intrinsic piezoresistive properties difficult to be used for enhancing the sensitivity of the sensors ${ }^{27,35}$. The need of the hour is finding cost-efficient methods of producing nanomaterial fillers that would facilitate further development, rapid prototyping, and subsequent commercialization of inexpensive yet highly sensitive and reliable devices for human motion monitoring applications ${ }^{19,36}$. Furthermore, the nextgenerations sensors must be skin-mountable noninvasive, lightweight, yet inexpensive to facilitate widescale applicability. All these requirements can be fulfilled by adapting a bio-inspiration approach. For instance, artificial skin-like sensors can pave the way for next-generation human motion monitoring, human-machine interface devices and myoelectric prostheses.

Skin is among the most sophisticated organs of our body responsible for somatosensory perception. In other words, skin can be considered as a complex large area pressure, tactile, temperature, and vibration sensor rivaling the complexity of auditory system. From the soothing touch sensation of silk to extreme painful sensation of finger prick or burn, skin is capable of discerning different kinds of noxious and innocuous stimuli. Underlying the hairy and glabrous skin (non-hairy) of mammals lie mechanosensory afferents and receptors which are responsible for the complex somatosensory abilities. A combination of Low threshold and High threshold mechanoreceptors (LTMRs/ HTMRs) innervate the skin, which facilitate sensing and response to both innocuous and noxious stimuli ${ }^{37}$. For instance, the glabrous skin of human hand, sole, and lips consist of LTMRs which can be classified into four distinct categories based on the type of stimuli they respond to and their firing interval and pattern. The slow adapting LTMRs (SA-LTMR) like Merkel cell and Ruffini endings respond to continuous indentation and skin stretch respectively while being relatively insensitive to objects moving across the skin. Slow adapting Ruffini endings enable the detection of finger positioning and shape which is also known as proprioception (sensation of positioning). On the other hand, rapidly adapting LMTRs (RA-LTMR) enable the detection of objects moving across the skin. The two different types of RALTMR in the glabrous skin, namely- Meissner Corpuscle and Pacinian corpuscle form a psychophysical frequency continuum capable of a detecting a broad range of frequencies in the range $\sim 10-300 \mathrm{~Hz}^{37}$. While the Meissner corpuscles are capable of detecting minutest of motions which enable hand to detect slipping of objects, the $\sim 2500$ oval shaped Pacinian corpuscles innervating our fingers enable to discern temporal structure of vibration ${ }^{37}$. Thus, in order to achieve true skin-like large area sensing capabilities, the desired sensors should be flexible, skinmountable, capable of detecting both static and wide bandwidth dynamic strain or tactile pressure stimuli.

In this work, we propose a facile method of developing a highly stretchable and ultralightweight skin-like CNF-PDMS compositebased strain/tactile sensors tailored for human motion monitoring applications. Electrospun polyacrylonitrile (PAN) nanofiber bundles were carbonized at a relatively low temperature $\left(950^{\circ} \mathrm{C}\right.$ in contrast to commercial CNFs pyrolyzed at $\sim 1300-2000^{\circ} \mathrm{C}$ ) to synthesize freestanding CNF membranes which were subsequently embedded into PDMS to fabricate strain and tactile sensors. The freestanding CNF bundles were studied employing a scanning electron microscope (SEM) to understand their morphology and conductive domain disconnection mechanism was invoked to explain their piezoresistive property. The fabricated CNF-PDMS strain and tactile sensors were characterized for quasi-static pressure stimuli response. From the quasi-static force-sensing tests, the response of the sensor was found to be linear for normal forces up to $20 \mathrm{~N}$, and the sensitivity of the sensor was determined to be $1.82 \mathrm{kN}^{-1}$. Reliability tests were conducted on the pressure sensor through cyclic loading involving at least 300 load cycles to demonstrate its overall durability. Furthermore, to demonstrate the vibration sensing property of the glabrous skin enabled by the Pacinian corpuscles, the pressure sensor responses were characterized for a series of oscillatory tactile pressure stimuli in the range $\sim 10-610 \mathrm{~Hz}$. To demonstrate the versatility of the proposed fabrication method and their potential for apparel integrability, various configurations of devices including a pressure sensor, a strain sensor, a 16-point pressure-sensitive, flexible sensor array, a stitchable strain sensor, and a gesture-tracking smart glove were demonstrated. In particular, the proprioceptory capability of the sensors were demonstrated by a gesture tracking smart glove capable of detecting at least 14 different unique gestures. Finally, the application of these sensors in monitoring real-time human motion was validated through experiments that demonstrate the broad applicability of such sensors in various applications including personalized health monitoring, soft robotics, myoelectric prosthesis and other wearable devices. In addition, a smart system comprising of five identical strain sensors mimicking proprioceptors and tactile sensors mimicking SA-LTMRs of skin is conceptualized which has potential for next-generation myoelectric prosthetic skins. Such apparel integrable sensors like the one demonstrated in this work will facilitate the development of a future generation of sophisticated yet aesthetically appealing smart apparel capable of tracking human movement in real-time (Fig. 1).

\section{RESULTS}

\section{Analyses to study the synthesis and piezoresistive nature of CNFs}

Electrospun PAN nanofibers were pyrolized at $950{ }^{\circ} \mathrm{C}$ to synthesize CNF bundles used for fabricating the sensors reported in this work. The details of processing conditions involved in electrospinning of PAN nanofibers as well as the pyrolization process to form CNF nanofibers were reported in our earlier works and are described in the "Methods" section ${ }^{38,39}$. In our previous work, a series of material characterization experiments involving X-ray photoelectron spectroscopy (XPS), Raman spectroscopy, and scanning electron microscope (SEM) imaging were conducted on the pyrolyzed nanofibers to understand the origin of piezoresistivity in the CNF bundles ${ }^{38}$. As evident from the micrographs in Fig. $2 a, b$ the diameters of the nanofibers were observed to have shrunk by $\sim 60 \%$ when pyrolyzed at $950{ }^{\circ} \mathrm{C}$, signifying weight loss due to the breaking of carbon-oxygen and carbon-nitrogen bonds (present in the PAN nanofibers) subsequently releasing oxygen and nitrogen, leading to the densification of the nanofibers due to the formation of carbon-carbon bonds. In our previous work ${ }^{38}$, the deconvolution analysis of C1s peak of XPS spectrum (not shown here) for the CNF bundle (pyrolyzed at $950^{\circ} \mathrm{C}$ ) strongly indicated the dominance of $\mathrm{sp}^{2}$ bonded carbon atoms. The results of the material characterization experiments clearly showed the carbonization in the electrospun nanofiber bundles at a relatively low temperature of $950{ }^{\circ} \mathrm{C}$. The presence of conductive $\mathrm{sp}^{2}$ hybridized carbon atoms in a non-conductive matrix of $\mathrm{sp}^{3}$ hybridized carbon (inferred from the characterization experiments in our previous work), explains the piezoresistivity observed in individual isolated $\mathrm{CNFs}^{38,40}$. 


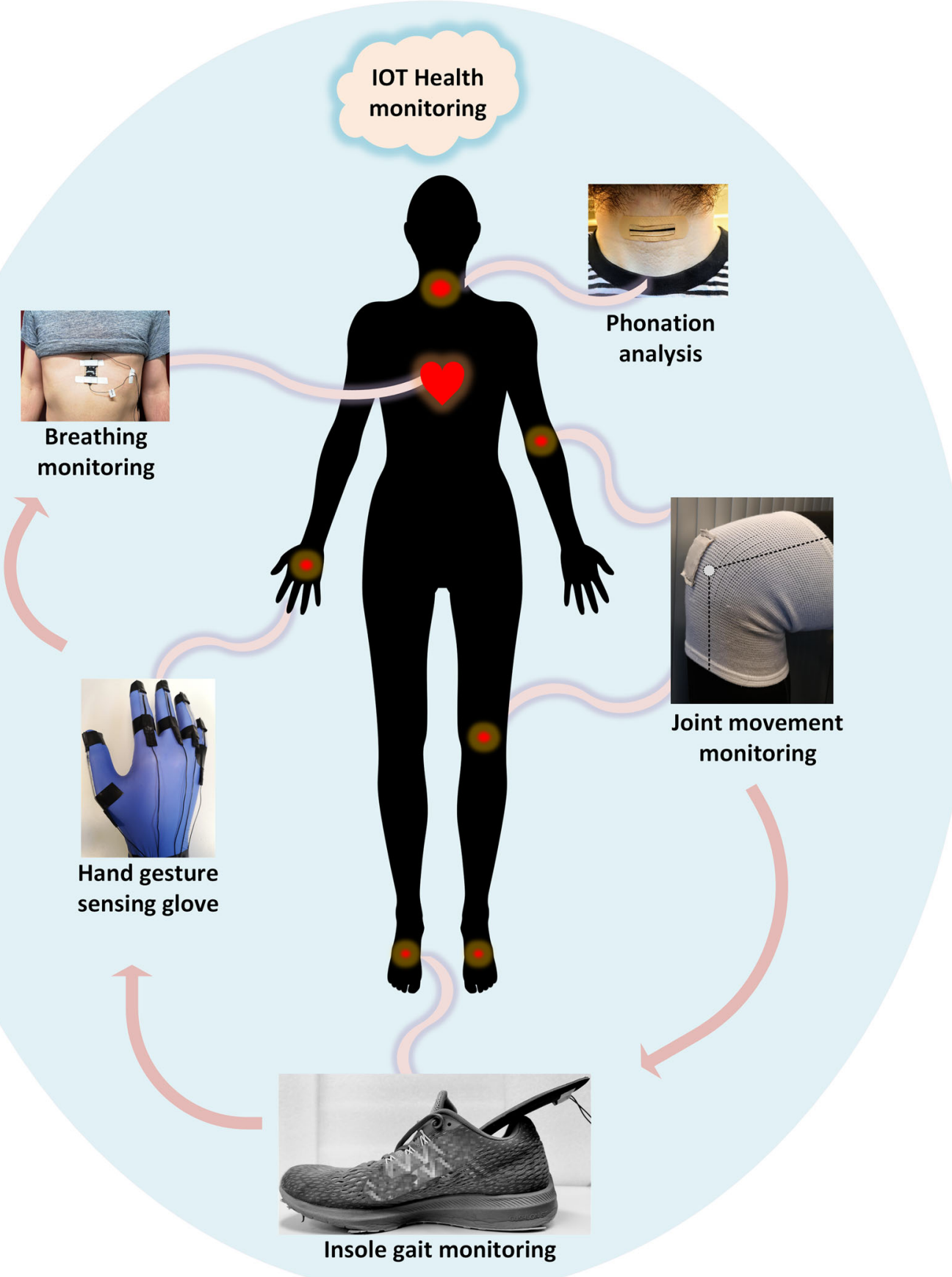

Fig. 1 Applications of CNF-PDMS sensors for human physiological locomotion monitoring. A schematic illustration of the human-motion monitoring, gesture recognition, and tactile sensing applications of the CNF-PDMS sensors demonstrated in this work.

In the past, researchers have hypothesized that the electrical conduction in CNFs takes place due to the electron mobility within the graphitic domains along with the inter-domain electron tunneling ${ }^{38,40}$. Based on the conductive domain discontinuity piezoresistivity model for carbonized nanofibers proposed by Cai et al. $^{40}$, the conductive $\mathrm{sp}^{2}$ hybridized regions (turbostratic domains) are embedded in non-conductive $\mathrm{sp}^{3}$ hybridized amorphous carbon atom matrix (schematically represented in Fig. 2c). Previously, Cai et al. proposed an equivalent electrical circuit model where the $\mathrm{sp}^{2}$ hybridized conductive regions represented by gray-colored boxes are in series with the nonconductive $\mathrm{sp}^{3}$ hybridized amorphous carbon atom matrix represented by resistors (schematically represented in Fig. 2 d) ${ }^{40}$.
The electron tunneling resistance is quite sensitive to the electron tunneling distance which leads to its high sensitivity towards local strain which explains enhanced piezoresistive performance in CNFs in comparison to carbon fibers having continuous turbostratic domain (turbostratic carbon refers to a class of carbonaceous materials having structural ordering lying in between that of $\mathrm{sp}^{2}$-hybridized graphite with stacked structure and $\mathrm{sp}^{3}$-hybridized amorphous carbon ${ }^{41}$ ). As the electrical conductivity in the nonconductive $\mathrm{sp}^{3}$ hybridized amorphous carbon region is mainly due to the tunneling effect, any strain resulting from applied mechanical load leads to a change in resistance owing to the change in tunneling distance. From the conductive domain discontinuity model explaining the piezoresistivity in CNFs, 
(a)

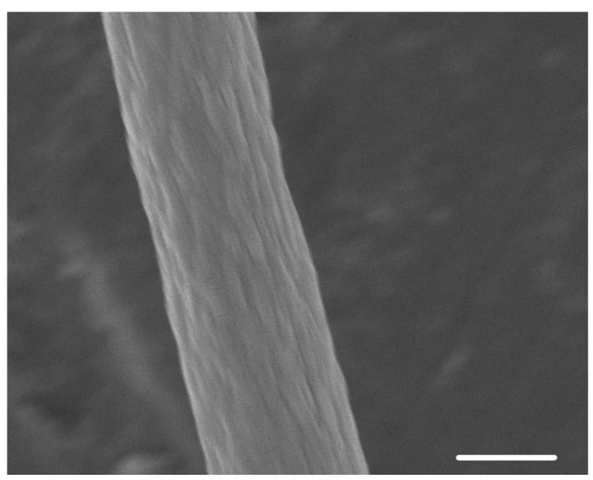

(b)

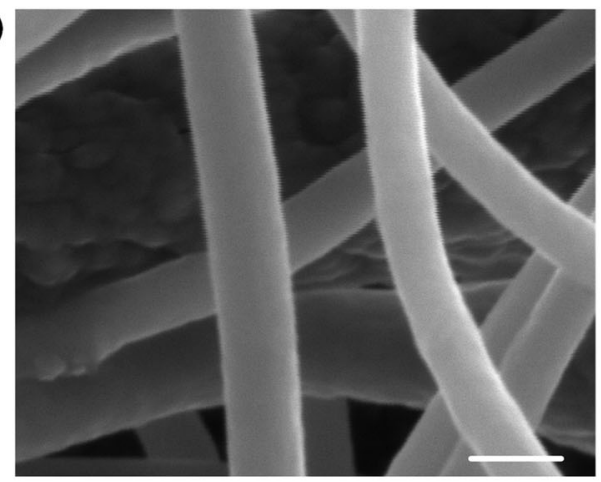

(c)

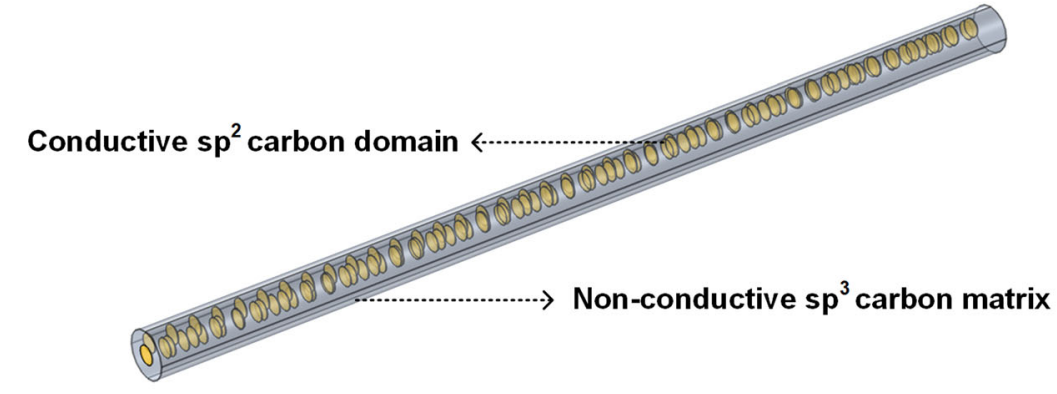

(d)

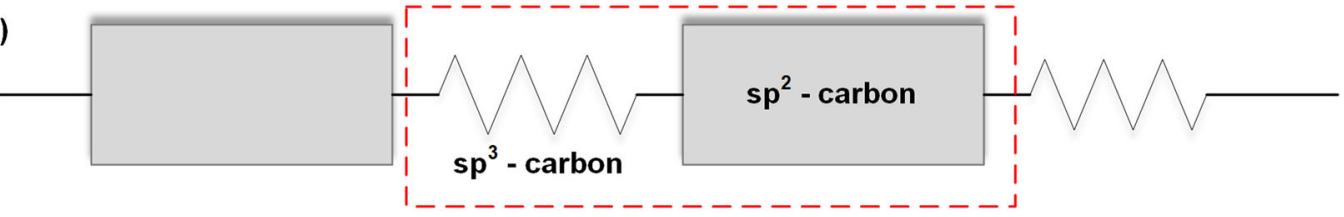

Fig. 2 Morphology of CNF bundle and sensing mechanism. a, b SEM micrographs showing the comparison between the diameters of the as-spun and pyrolized nanofibers. c A 3-d model of an isolated electrospun CNF. Gold-colored plates represent the conductive sp ${ }^{2}$-hybridized carbons in a non-conductive matrix (gray-colored) of $\mathrm{sp}^{3}$-hybridized carbon atoms. $\mathbf{d}$ Electrical equivalent model of an isolated single CNF. Scale bars: $\mathbf{a}, \mathbf{b}=500 \mathrm{~nm}$.

it can be argued that in our case, the PAN nanofibers annealed at $950^{\circ} \mathrm{C}$ should show piezoresistivity owing to graphitization which is evident from the Raman spectroscopy and other material characterization results as established in our previous work $^{38}$. In the past, a significant amount of research has been conducted towards understanding the charge transport mechanisms in nanofiller-polymer composites. Two main mechanisms identified were contact conductance between the nanofillers, and electron tunneling between two conductive nanofillers in close proximity separated by an insulating barrier ${ }^{42-44}$. Simmons et al. formulated a series of equations to predict the tunneling effect between two conducting electrodes separated by an insulating polymer potential barrier of arbitrary shape ${ }^{43}$. Furthermore, Ounaries et al. experimentally studied the conduction characteristics of single walled carbon nanotube (SWCNT) polyimide composites and reported non-ohmic current-voltage characteristics strongly suggesting a quantum mechanical tunneling phenomenon ${ }^{45}$. In the case of a CNF bundle embedded in a polymer/elastomer matrix, strain-sensitive resistance change mechanism could arise from the conductive domain disconnection mechanism which has been widely reported in the past for other nanomaterial-polymer composites ${ }^{10,11,46,47}$. Within the CNFs embedded in a polymer matrix, the electrons pass through the overlapping network of conductive nanofibers. When an external force/stress is applied, it causes a change in the overlapping area between adjacent nanofibers thus leading to a change in the overall resistance of the bundle. Also, electrons can tunnel across a thin polymer barrier separating two adjacent nanofibers, thus forming quantum tunneling junctions. The tunneling resistance between two adjacent CNFs separated by a polymer layer can be predicted using Simmon's tunneling resistance theory ${ }^{43,44}$.

\section{Sensor fabrication and characterization}

For different applications, various designs of sensors suitable for pressure or strain sensing were fabricated. Figure 3a schematically shows the steps involved in the fabrication of the CNF-PDMS strain sensor. More details of the device fabrication steps are provided in the "Methods" section. Figure 3b shows the image of the fabricated flexible strain sensors. The strain sensor was stretched to at least $50 \%$ of its original length to demonstrate its stretchability as shown in Fig. 3c.

A study conducted by Asakawa et al. ${ }^{48}$, investigated the range of force applied by fingers of thirteen individuals using touchscreen devices. The study reported mean force values of $0.5 \mathrm{~N}$ for tapping, $0.79-1.18 \mathrm{~N}$ for sliding gestures, and $2.05 \mathrm{~N}$ for stretch. Although average human beings can exert force much higher than $50 \mathrm{~N}$ with their fingers, for the sake of evaluating tactile sensing performance of the CNF-PDMS sensor fabricated in this work, a tactile force range of $0-20 \mathrm{~N}$ was chosen. To assess the strain/tactile pressure sensing performance of the CNF-PDMS sensor, a series of tests involving quasi-static tactile pressure sensing were performed. As the sensor developed in this work is intended for use as tactile sensor for wearable applications, a touch emulation experiment was conducted to evaluate the performance of the sensor. The experimental setup to assess the quasi-static tactile pressure sensing performance comprised of a PDMS finger model molded from a human finger secured on the movable piston of Instron 5940 universal testing system (schematically represented in Fig. 4a). Details of the test setups 
(a)
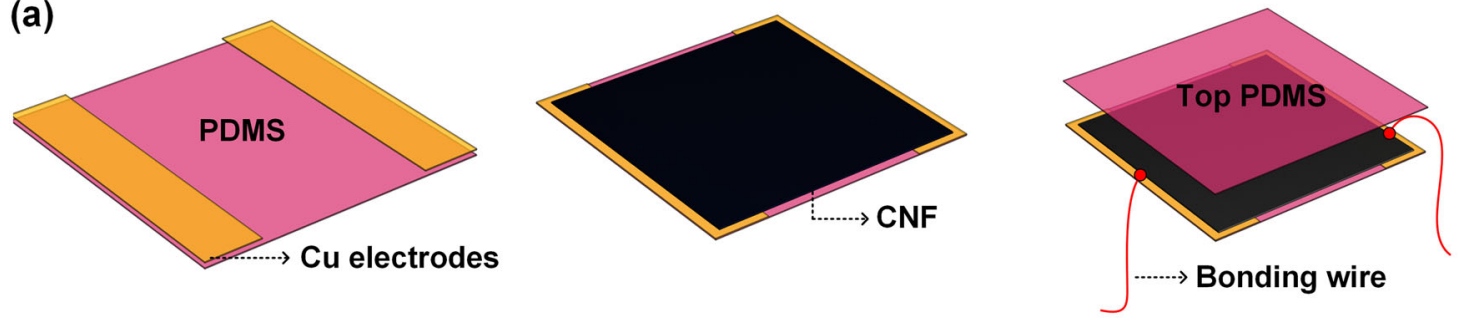

(b)

(c)

Relaxed state

Stretched $50 \%$
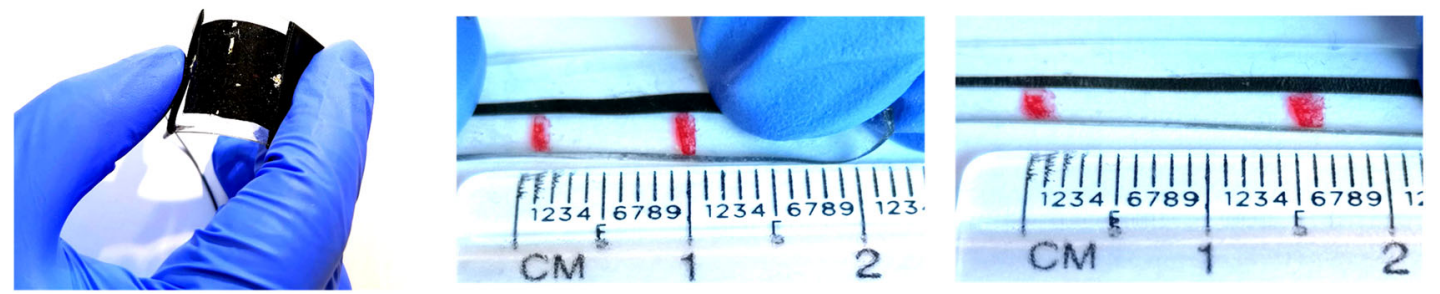

Fig. 3 Flexible strain sensor. a Schematic representation of the process steps involved in the fabrication of the carbon nanofiber-based strain sensor. b Photograph of the fabricated flexible CNF-PDMS strain sensors. c CNF-PDMS strain sensor in relaxed and stretched (50\% strain) condition.

are provided in the "Methods" section. A pre-compressive load of $\sim 640 \mathrm{mN}$ was applied before commencing the tests. For determining the force sensitivity, the applied force was varied from 0 to $20 \mathrm{~N}$ in steps of $666 \mathrm{mN}$ and the experiment was repeated 7 times as shown in Fig. 4b. From the bridge output, normalized change in resistance versus the applied force was plotted with standard deviation (Fig. 4c). The offset due to precompression load was reset to 0 in the post processing steps to determine the relative change in resistance. It was observed that, the sensor showed an overall resistance change of $\sim 3.6 \%$ upon application of $20 \mathrm{~N}$ force. As it can be seen in the plot in Fig. $4 \mathrm{~b}$, the response of the sensor was found to be linear for normal forces up to $20 \mathrm{~N}$ and the sensitivity of the sensor was determined to be $1.82 \mathrm{kN}^{-1}$. As observed from the results of the force calibration experiments, the sensor behaves in accordance with the conductive domain discontinuity explained earlier. A localized pressure/stress causes displacement of the nanofibers within the polymer matrix leading to a change in overlap area of the nanofiber percolation network which is subsequently manifested as resistance change response. In order to assess the reliability of the sensor over prolonged usage, the sensor was also subjected to an accelerated lifetime test comprising of a series of loadingunloading cycles at three different forces $4.5 \mathrm{~N}, 12 \mathrm{~N}$, and $20 \mathrm{~N}$ (comprising of 100 cycles each). The normalized relative change in resistance during the successive cycles was recorded and the offset due to pre-compression was set to zero to determine the overall relative change in resistance. The load curves were plotted along with the sensor response curves (Fig. $4 d-f$ ) and no noticeable delays were observed between the loading stimuli and sensor response. In our previous work, quasi-static tensile strain sensing experiments were conducted to evaluate the gauge factor of the CNF-PDMS sensor ${ }^{38}$. The sensor demonstrated a linear response with an average gauge factor of $\sim 11.14$ in response to tensile strain over a range of $0-50 \%$.

\section{Sensor applications in human motion monitoring}

One of the hallmark features of the SAll-LTMRs is their sensitivity to skin stretch. For instance, the SAll-LTMRs in human hand share certain physiological traits with proprioceptors which facilitate detection of finger conformation or proprioception ${ }^{37}$. Skin-like flexible large area sensors capable of detecting strain are not only suitable for rendering kinesthetic sense in prosthetic limbs and robotic arm but also for non-invasive monitoring of human motion parameters and vitals.

Continuous human motion monitoring can enable early diagnosis of diseases such as strokes, multiple sclerosis, and Parkinson's disease and assist physicians and physiotherapists dealing with such patients towards develop personalized treatment plans. To demonstrate the applicability of the CNF-PDMS sensors in kinesthetic perception and human motion monitoring applications, various human gait experiments involving wristmovement detection, walking, jogging, and respiration monitoring were conducted. In the first experiment, the sensor's response to wrist movements (both bending and side flicking) was determined by securing the sensor on the wrist of the left hand of a human subject. The test subject was directed to bend his wrist in controlled fashion to $30^{\circ}, 45^{\circ}$, and $90^{\circ}$ (in an arbitrary order; the bending angles have been approximated). It can be seen from the sensor response (Fig. 5a) that the angle of bending of the wrist could be clearly resolved by observing the output peak intensities in response to bending. Large wrist bending generated more intense peaks in comparison to the smaller ones with $90^{\circ}$ bending consistently generating the largest response peaks. To understand the effect of sideways movement of the wrist on the response, the sensor was secured on the left hand of a human subject and the response of the sensor to stimuli involving flicking the wrist left and right is shown (Fig. 5b). Left-ward flicks consistently produced more intense signal as compared to the right-ward flicks. The consistent mismatch of signal intensities while flicking the wrist left and right directly corresponds to the person's maximum achievable bending angle towards each direction. To demonstrate the capability of the sensor in detecting respiratory breathing patterns, the sensor was secured on the diaphragm region at the base of the chest of a person as shown in Fig. $5 \mathrm{~d}$. Responses of the sensor towards normal and deep breathings were acquired as shown in Fig. $5 c$, d. Clear differentiation between various breathing patterns could be achieved by observing the signal intensity and frequency. The next experiment involved the recognition of the gait pattern of a human foot during various motions such as walking and jogging. A strain sensor was secured on the heel of a human subject and the sensor responses were acquired for walking and spot jogging as shown in Fig. $5 e, f$ respectively. As observed from the plots the signal peak intensities and frequencies provide information of the gait of a person. While the sensor demonstrated blunter peak towards walking, 
(a)

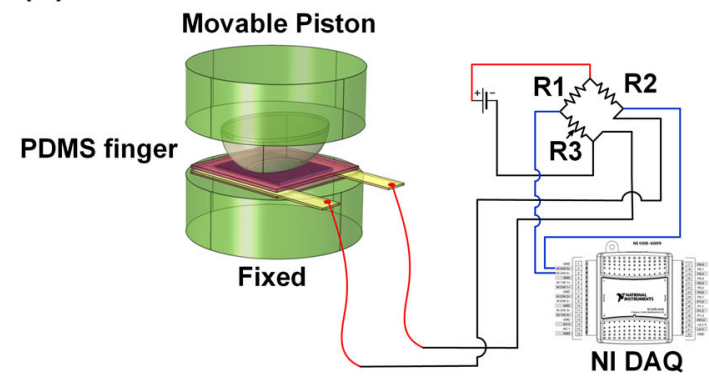

(c)

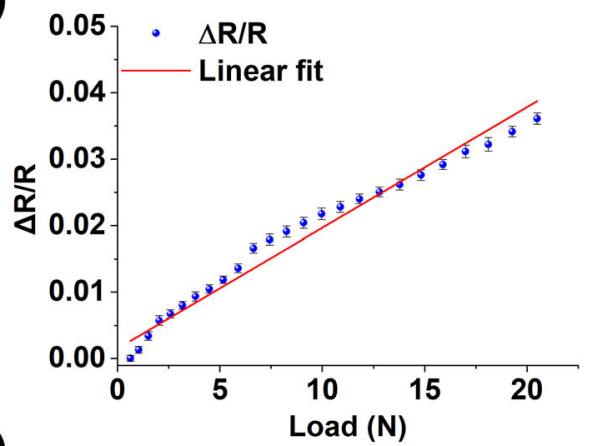

(e)

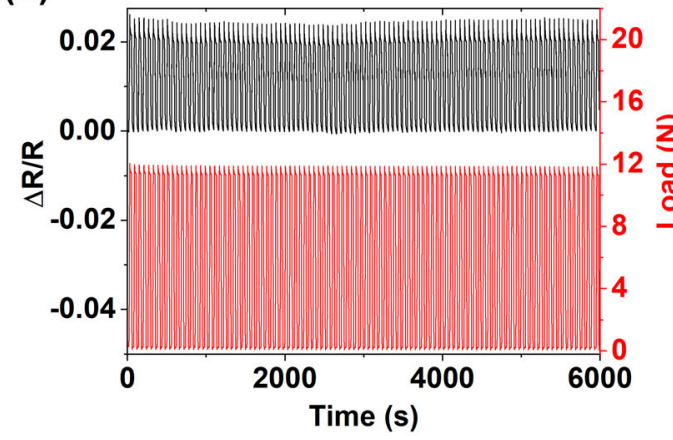

(b)

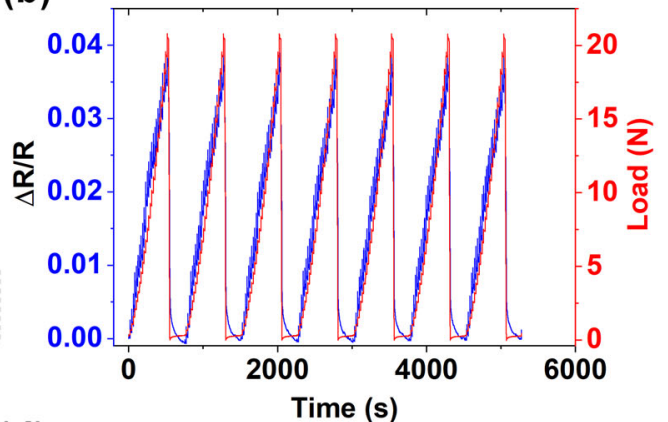

(d)

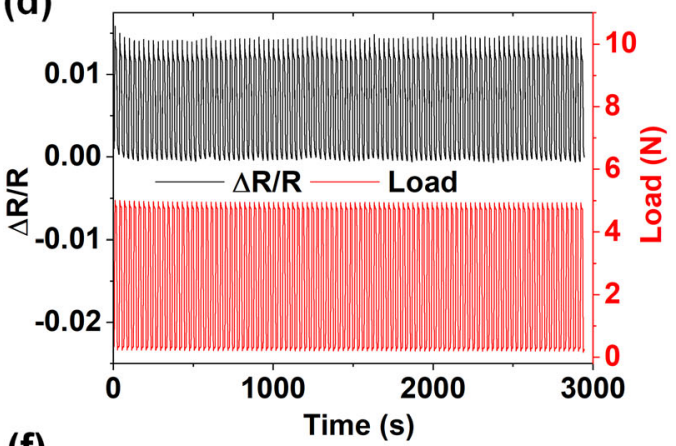

(f)

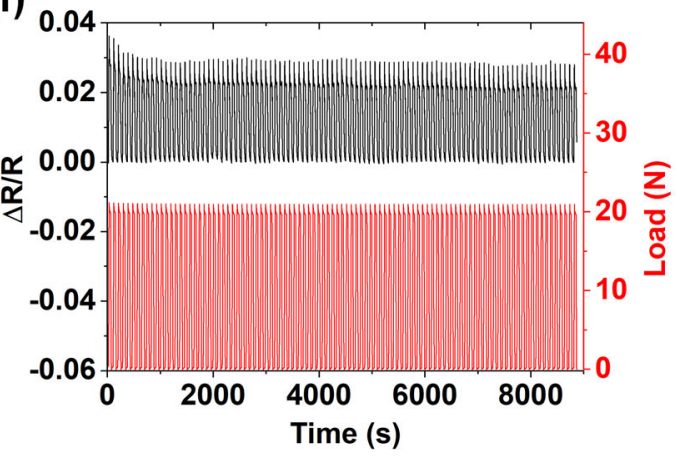

Fig. 4 Flexible strain sensor characterization. a Schematic representation of the experimental setup used for the characterization experiment. b Plot showing loading versus normalized resistance change (repeated seven times). c Plot showing the normalized resistance change versus the applied force with the error bars representing standard deviation (s.d.). d-f Response of the sensor to cyclic loading at three different forces- $4.5 \mathrm{~N}, 12 \mathrm{~N}$, and $20 \mathrm{~N}$ (100 cycles each).

the response towards jogging demonstrated very sharp and intense peaks signifying the capability of the sensor to distinguish between different movement patterns encountered daily. Supplementary video $\mathrm{V} 1$ shows real time gait signal acquired from the right foot of a test subject.

\section{Gesture tracking smart glove}

Gesture detection can often be useful for applications involving robotics-assisted surgery, robotics-assisted bomb disposal, robotics rehabilitation and other tasks demanding precise robotic control with human input. In order to further demonstrate the proprioceptive capability of the CNF-PDMS strain sensors, five stretchable strain sensors were assembled and secured onto a nitrile glove (Fig. 6a, b), and real-time finger gesture-sensing experiments were conducted. All the individual sensors on the smart glove were connected to appropriate Wheatstone bridge circuits for real-time data acquisition. Each of the five fingers of the right hand starting with the thumb finger was flicked once. The sequence was repeated thrice and the sensor outputs from the corresponding Wheatstone bridges were logged individually as shown by the plot in Fig. 6c. The raw signal outputs acquired from each of the five fingers of the right hand were then represented by employing a color grid plot where each individual grid of a particular row corresponds to the signal value acquired from the corresponding finger at a particular point of time as shown in Fig. 6d. Supplementary video V2 shows the real time signals acquired from the middle and ring finger of a test subject. As observed in the video, the ring and middle fingers show mild crosstalk. While the middle finger is moved in a steady and controlled fashion, the adjacent fingers (namely, index and ring fingers) also demonstrate mild movements which are picked up by the sensitive sensors on the gesture tracking smart glove. This information can be valuable for developing sophisticated systems for remote robot-assisted precision operations with human inputs where the system can accurately mimic natural movement of human fingers (where it is natural to observe mild tremors in the adjacent fingers of a particular finger which is moving).

Furthermore, a more comprehensive evaluation test comprising of 14 different finger gestures was conducted to demonstrate the usability of the smart glove device. Figure 7 shows the summary of 14 different hand gestures which were implemented with the smart glove and represented using a color grid-plot scheme for easy visualization. We start with all the fingers in relaxed position. 
(a)

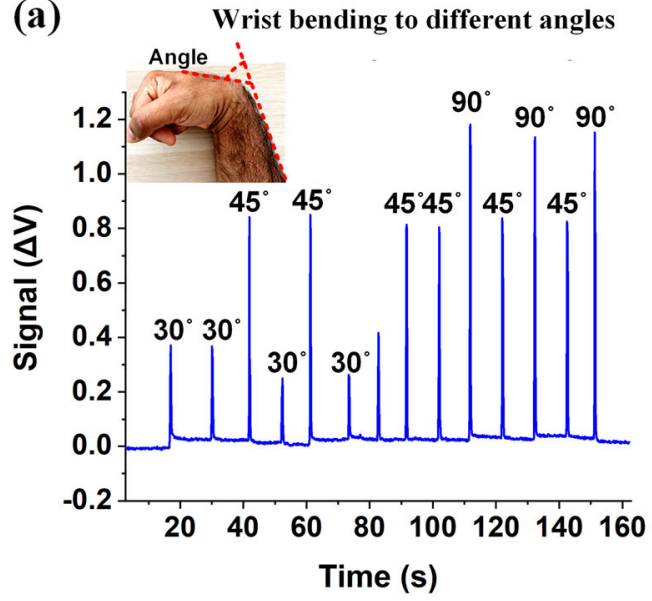

(c)

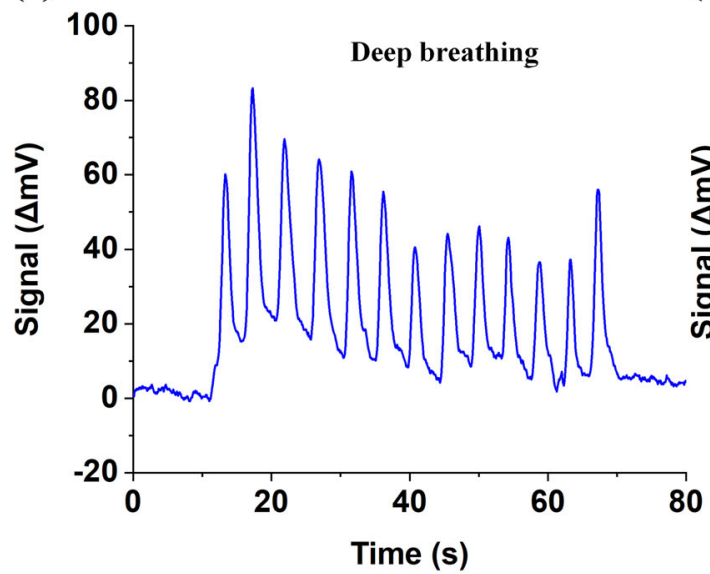

(e)

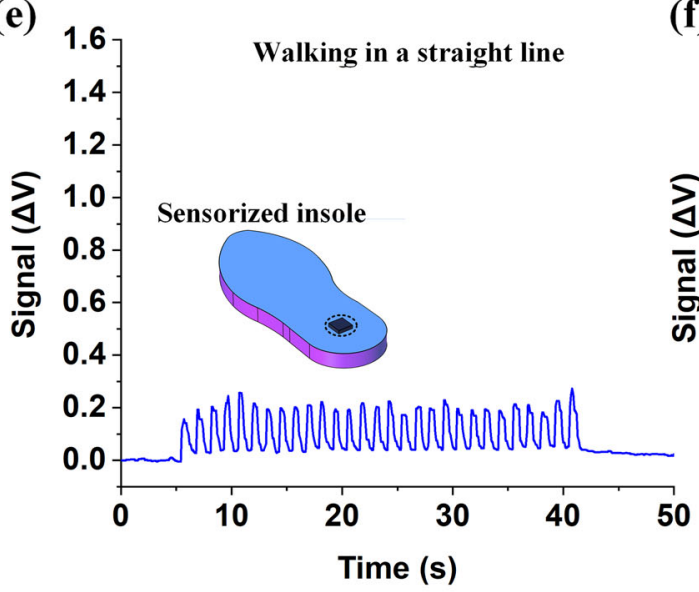

(b) Wrist bending left and right

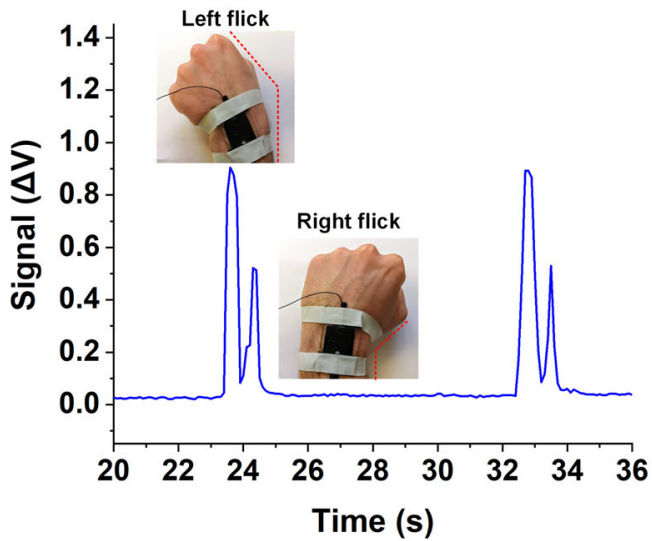

(d)

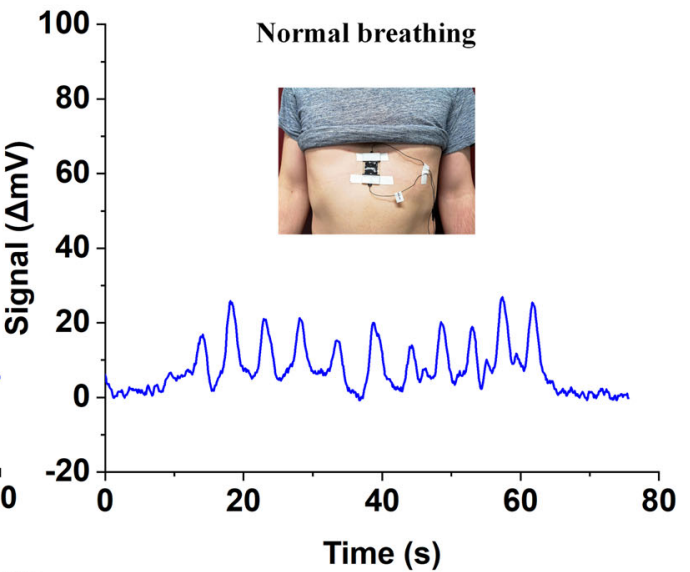

(f)

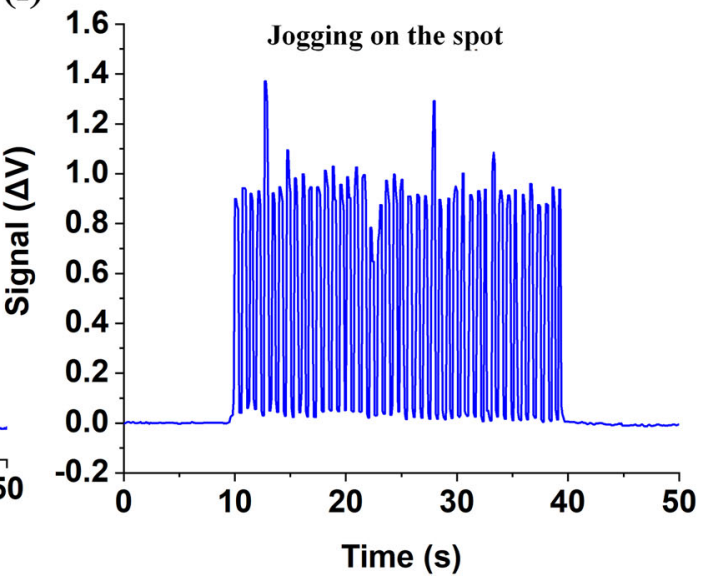

Fig. 5 Application of the flexible strain sensor in human motion monitoring. a Response of the sensor to various wrist bending angles. b Response of the sensor to sideways wrist flicking. $\mathbf{c}, \mathbf{d}$ Response plots of the sensor to deep and normal breathing. e, $\mathbf{f}$ Response of the sensor to walking in a straight line and spot jogging, respectively.

Gestures "Flick 1-5" represent individual finger flicking of all the five fingers of the right hand starting with the thumb. Then, we continue to demonstrate 14 different gestures using unique combinations of the five finger-positions which are reflected by the color plot generated from the raw signals acquired from the smart glove with accompanying Wheatstone bridge circuit setup. The results from the gesture sensing experiments clearly demonstrate the potential of the CNF-PDMS sensor for wearable skin and soft robotics applications.

\section{Apparel integrable and stitchable sensor}

The fabric-like texture, flexibility, and stretchability of the CNFPDMS sensor also allowed simple integration of the sensors into a wearable fabric-like materials for apparel integration. To demonstrate the feasibility of including the sensor in apparel integrable applications, a sports kneecap featuring a CNF sensing element was developed and tested. Figure 8a schematically shows the steps involved in the fabrication of the apparel integrable sensor. First, a sheet of CNF bundle having the dimensions of $2 \mathrm{~cm} \times 2 \mathrm{~cm}$ 
(a)

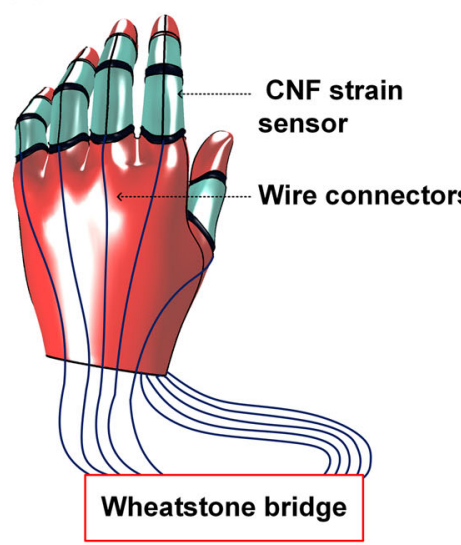

(b)

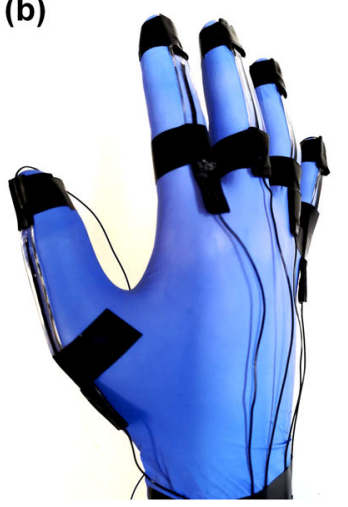

(c)
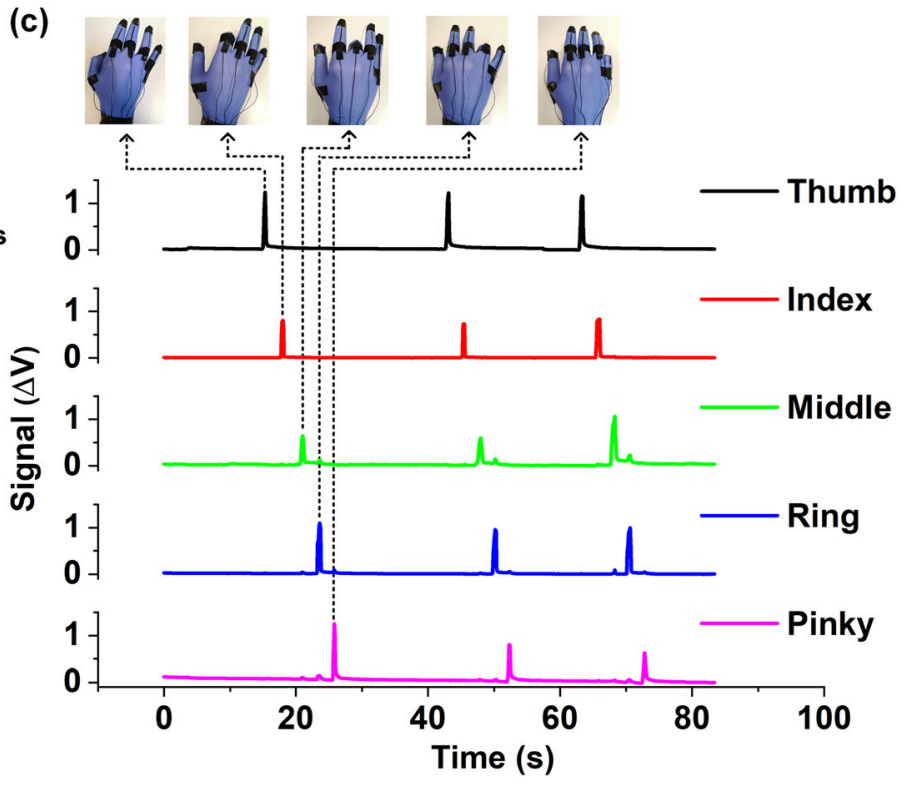

(d)

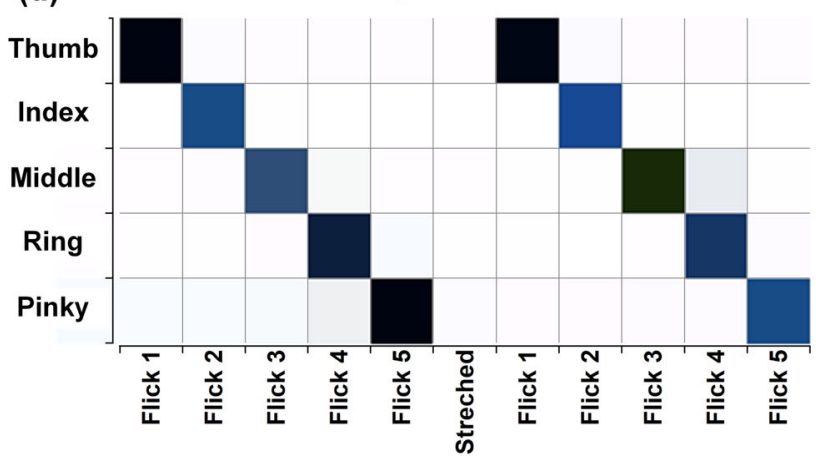

Signal $(\Delta \mathbf{V})$

1.250

0.8333

0.4167

0.000

Fig. 6 Gesture-sensing smart glove. a Schematic representation of the gesture-sensing smart glove comprising of the individual five finger movement sensors. b Photograph of the gesture-sensing smart glove worn for demonstration. c Plots showing the response of individual fingers on the smart glove starting with thumb. $\mathbf{d}$ Color grid-plot demonstrating the sensor outputs for the individual finger movements.

was stitched to a base cotton cloth layer. The CNF bundle was then bonded to two copper tapes on the two edges using conductive silver epoxy. Another layer of cotton cloth was stitched on the top to completely cover the nanofibers. Finally, the gait sensing functionality of the sensor stitched on the wearable kneecap was demonstrated by making a test subject wear the device and bend his knee (up to $90^{\circ}$ ) in a controlled fashion staring from a straight and relaxed state. As observed from Fig. 8b, depending on the speed of bending the knee, the signal width varied which can be useful for precisely tracking knee movements during performance sports such as sprint, football, and other similar activities.

\section{Fabrication and testing of a multi-point touch sensitive flexible sensor array}

Physiological properties of SAI-LTMRs of skin render them the ability to encode highly complex spatial image of tactile stimuli $i^{37}$. Skin-like sensors having the capability of mapping multiple pressure/tactile stimuli points over a two-dimensional plane are desirable for mimicking the sense of touch. To further demonstrate the applicability of the CNF sensors in myoelectric prosthesis, soft robotics, and artificial electronic skins, we have also demonstrated a large area multi-point touch sensitive flexible tactile sensing array using the CNFs. Figure 9a shows the schematic representation of the process steps involved in developing the transparent multi-point touch sensitive flexible sensor array. For the purpose of representation, the process steps involved in the fabrication of a 25 -points pressure sensitive flexible sensor array have been demonstrated in Fig. 9a. Five, $2 \mathrm{~mm}$ wide and $10 \mathrm{~cm}$ long strips of electrically bonded CNF bundles were placed in parallel ( $1 \mathrm{~cm}$ apart from each other) on a cured PDMS film. The first layer was then covered with PDMS and cured. Five similar electrically bonded carbon nanofibers strips were then placed on top of the first layer in an orthogonal fashion. Finally, the second layer was covered with PDMS and cured to achieve a complete encapsulation. The actual fabricated device has four strips of carbon nanofiber bundles in each of the two layers which combine to form 16 pressure sensitive points as shown in Fig. 9b. The proposed design/fabrication process is scalable and can be extended to include more touch sensitive points. Figure $9 c$ shows the schematic representation of the working mechanism of the multi-point touch sensitive flexible sensor array. The device consists of 8 sensor lines numbered alphabetically $(A-H)$. The individual sensors in the group $A-D$ and $\mathrm{E}-\mathrm{H}$ are aligned in parallel to each other respectively, while the group $A-D$ is orthogonal to $E-H$ as depicted in Fig. 9c. Each intersection of two sensor lines corresponds to one touch sensitive point in the multipoint array. Hence, 8 sensors lead to 16 touch sensitive points, which can be identified as a combination of signal outputs from two lines. 
(1)

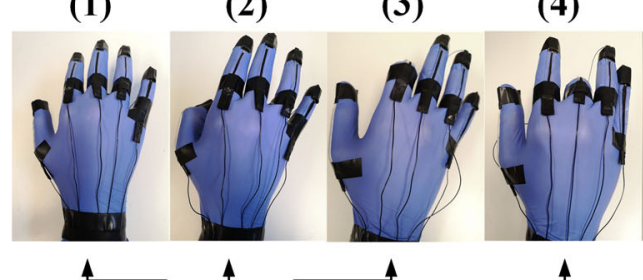

(5)
(6)
(7)
(8)
(9)

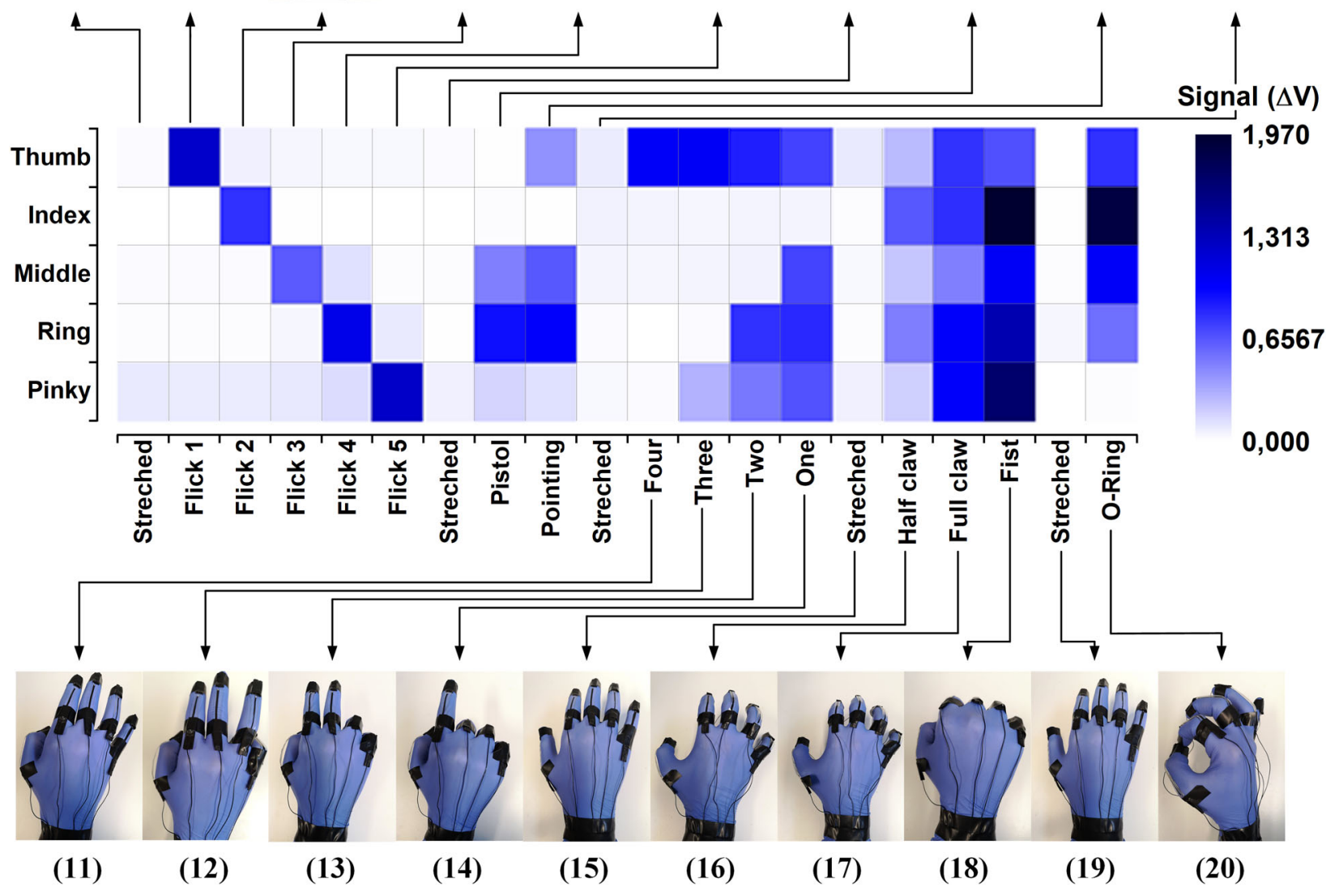

Fig. 7 Gesture-sensing smart glove. Color grid-plot showing the capability of the smart glove in detecting different hand gestures represented by unique combinations in the color-grid plot.

In order to test the functionality of the array, each point on the flexible sensor array was pressed lightly with the tip of the finger as the responses of all the sensors in the array were acquired. First, all points along sensor line $A$ were tested from the intersection starting at $\mathrm{A} \times \mathrm{E}$ and ending at $\mathrm{A} \times \mathrm{H}$. Likewise, the lines $B, C$, and $D$ were tested in the same fashion. The order in which all the points on the flexible sensor array were tested is summarized by the table in Fig. 9c. The resistance change induced by the touch, resulted in a change of voltage that could be read using a Wheatstone bridge circuit accompanying each of the sensors. A color grid plot summarizing the signal reception from all the individual points on the flexible sensor array was developed. Each individual box in the color grid plot graphically represents the change in voltage output of the Wheatstone bridge circuit corresponding to the sensor line being pressed/ touched. Figure $9 c$ graphically represents the change in Wheatstone bridge output at the instance when an individual point in the flexible sensor array was touched. For example, when point "P1" on the flexible sensor array is pressed, the grid boxes (in the color plot) corresponding to sensor lines ' $A$ ' and ' $E$ ' show the appropriate voltage change (acquired from the corresponding Wheatstone bridge circuits) by means of a color scheme thus signaling the particular point being touched or pressed. Similarly, when point "P16" is pressed, the grids corresponding to sensor line " $\mathrm{D}$ " and " $\mathrm{H}$ " display the appropriate voltage changes. The simple application of identifying the local position of press/touch, was carried out by applying individual appropriate threshold values to analog signal outputs from the Wheatstone bridges corresponding to all the individual sensor lines (more details regarding the thresholding algorithm is furnished in the Supplementary information file). Individual thresholds were necessary for the proposed prototype since the fabrication process resulted in slightly different shapes of the sensor lines $(\mathrm{A}-\mathrm{H})$ leading to each line having a different sensitivity towards touch, implying a varying range of voltage change upon being touched/pressed. Furthermore, a threshold was necessary to remove false signals from the reading. After processing the data, false undesired signals were removed and only the intersecting lines corresponding to a specific point being pressed/touched displayed a value of 1 represented by red color in the color-grid plot shown in Fig. $9 \mathrm{~d}$. With the current design decision of choosing two intersecting sensor lines to form a sensing pixel, it is not possible to accurately discern between multi-point pressure stimuli applied simultaneously (more discussion is furnished in the Supplementary information file). The drawback can be mitigated by implementing individual sensors for each of the sensing touch points. 
10 (a)

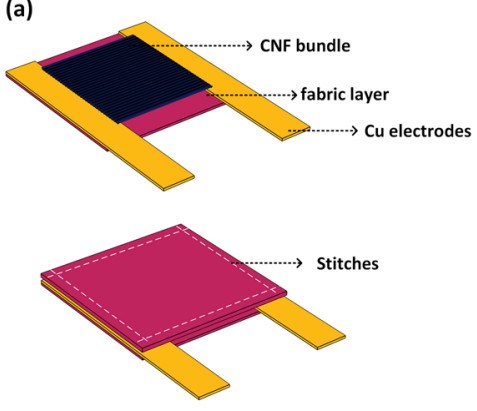

(b)

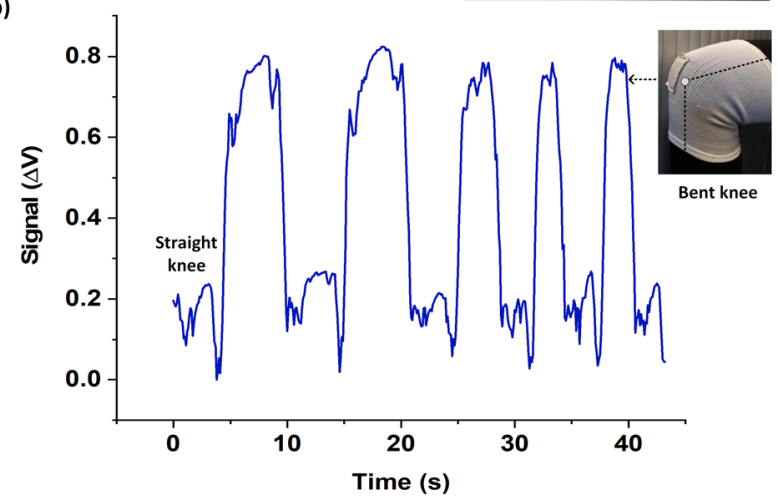

Fig. 8 Stitchable apparel integrable sensor. a Schematic representation of the fabrication steps involved in the development of the apparel integrable strain sensor. b Plot demonstrating the functionality of the sensor stitched into a sports kneecap.

\section{Conceptual artificial skin for recreating the sense of touch}

Skin is one of the most sophisticated and reliable natural sensors which enables somatosensory perception. Figure 10a (adapted with permission from Cell press ${ }^{37}$ ) shows a schematic representation of the anatomy of glabrous skin and it's underlying mechanoreceptors as discussed previously. Though the CNF PDMS stretchable sensors developed in this work is intended for human motion monitoring applications, an integrated system comprising of the gesture sensing smart glove and the multi point touch sensitive array can be combined with a custom designed neuromorphic circuit to mimic human hand as shown in the conceptual schematic in Fig. 10b. As discussed previously, the strain sensors configured on the fingers represent proprioceptors capable of detecting bending thus facilitating kinesthetic perception. For instance, the gesture tracking glove can clearly discriminate between the fingers in a relaxed position vs "O-Ring" gesture as demonstrated by the real-time sensor response plot in Fig. 10b.

Whereas, the tactile sensor array integrated on the palm region of the glove would be capable of identifying tactile stimuli and object orientation. Also, to mimic the vibration detection ability of the RA-LMTRs (Meissner and Pacinian corpuscles), the CNF-PDMS tactile sensor was a subjected to a series of cyclic tactile stimuli in the frequency range $10-610 \mathrm{~Hz}$ (details of the vibration response test setup are provided in the experimental section). The response of the sensor to the vibrations is summarized by the stimuli-response matrix plot in bottom right of Fig. 10b. Device voltage response was normalized and plotted along with the input stimuli frequencies.

\section{DISCUSSION}

In conclusion, this work demonstrates the applicability of electrospun CNF bundles as sensitive, flexible, and linear yet inexpensive piezoresistive sensing element in developing apparel integrable sensors for wearable human motion monitoring and tactile sensing applications. The sensor demonstrated in this work utilized electrospun CNF bundles embedded in PDMS elastomer forming a nanofiber percolation network that responds to strain under the conductive domain disconnection mechanism. Various material characterization experiments conducted previously on the pyrolyzed nanofibers revealed the carbonization of the electrospun PAN nanofibers at a relatively low temperature of $950^{\circ} \mathrm{C}$. Morphological analyses were conducted on the freestanding CNF bundles employing an SEM, and the conductive domain disconnection mechanism was invoked to explain their piezoresistive property. Quasi-static normal force response characterization experiments conducted on the sensor also revealed a linear response with a force sensitivity of $1.82 \mathrm{kN}^{-1}$. In addition, response of the sensor over wide band $(10-610 \mathrm{~Hz})$ dynamic tactile pressure stimuli was demonstrated. To demonstrate the feasibility of applying the sensor for various wearable devices and personalized health monitoring applications, various real-time human motion monitoring experiments involving walking, jogging, breathing exercise, joint movements were conducted. A gesture-tracking smart glove and a 16-point touch sensitive flexible sensor array was fabricated and demonstrated to identify various hand gestures and 16 distinct pressure points respectively, thus consolidating the immense potential of the sensors for smartskin applications. Finally, a stitchable and apparel integrable sensor was demonstrated to track knee joint movements successfully. The sensing materials and the facile fabrication method of developing highly flexible, stretchable, and stitchable pressure and strain sensors demonstrated in this work could guide the development of several flexible, wearable and skin-mountable sensors in future for applications in the fields of smart fabrics, myoelectronics, soft robotics, health monitoring devices, and biomedical devices.

\section{METHODS}

\section{Electrospinning of PAN nanofibers}

For electrospinning of the PAN precursor nanofiber bundles, Polyacrylonitrile powder (MW 150,000 g/mol) and N, N-dimethylformamide (DMF) purchased from Sigma-Aldrich, the Netherlands were used. A 9\% (w/v) solution of the PAN powder in DMF was prepared. An electrospinning setup (NanoSpinner NE300) from Inovenso ${ }^{T M}$ electrospinner fitted with a stainless-steel rotating mandrel collector was used for electrospinning PAN nanofibers. A standard syringe pump fixed with a $10 \mathrm{ml}$ syringe was used to feed the polymer solution through a needle $(18 \mathrm{G})$ at a constant flow rate of $1 \mathrm{ml} / \mathrm{h}$. Electrospinning was conducted by applying $12 \mathrm{kV}$ between the needle and a rotating mandrel collector $(10 \mathrm{~cm}$ in diameter) spinning at $1500 \mathrm{rpm}$ with a gap of $15 \mathrm{~cm}$ between the two. The electrospinning process was carried out for $30 \mathrm{~min}$ to obtain a film of uniform nanofibers deposited on the aluminum foil substrate.

\section{Pyrolyzation of the PAN precursor}

The electrospun nanofiber mats were initially transferred from the aluminum foil substrate to a ceramic crucible for high temperature pyrolization process. For the first step involving stabilization to form a layer of oxide, the nanofibers were heat-treated at a temperature of $245^{\circ} \mathrm{C}$ for $60 \mathrm{~min}$ in the presence of ambient air. A constant ramp rate of $5^{\circ} \mathrm{C} / \mathrm{min}$ was used to ramp-up to the stabilization temperature. Following the stabilization, in order to conduct pyrolyzation, the tube furnace was sealed air tight with a constant nitrogen $\left(\mathrm{N}_{2}\right)$ purge for $30 \mathrm{~min}$ to drive out the air inside. Thereafter, the nanofibers were ramped up at a rate of $5^{\circ} \mathrm{C} / \mathrm{min}$ in $\mathrm{N}_{2}$ ambience to $750^{\circ} \mathrm{C}$ and held for $60 \mathrm{~min}$ followed by a final ramp-up at the previously mentioned rate to $950^{\circ} \mathrm{C}$ where it was plateaued for another $60 \mathrm{~min}$ before ramping down to the room temperature at the same ramp rate.

\section{Morphology of the carbon nanofibers}

FEI Nova NanoSEM 230 field-emission scanning electron microscope (FE-SEM) was employed to study the morphological properties of the 
(a) Step 1: First layer

Step 2: Isolation layer

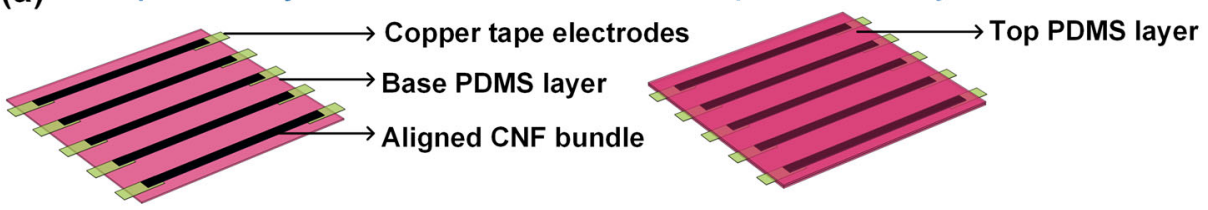

Step 3: Second layer

Step 4: Final Encapsulation
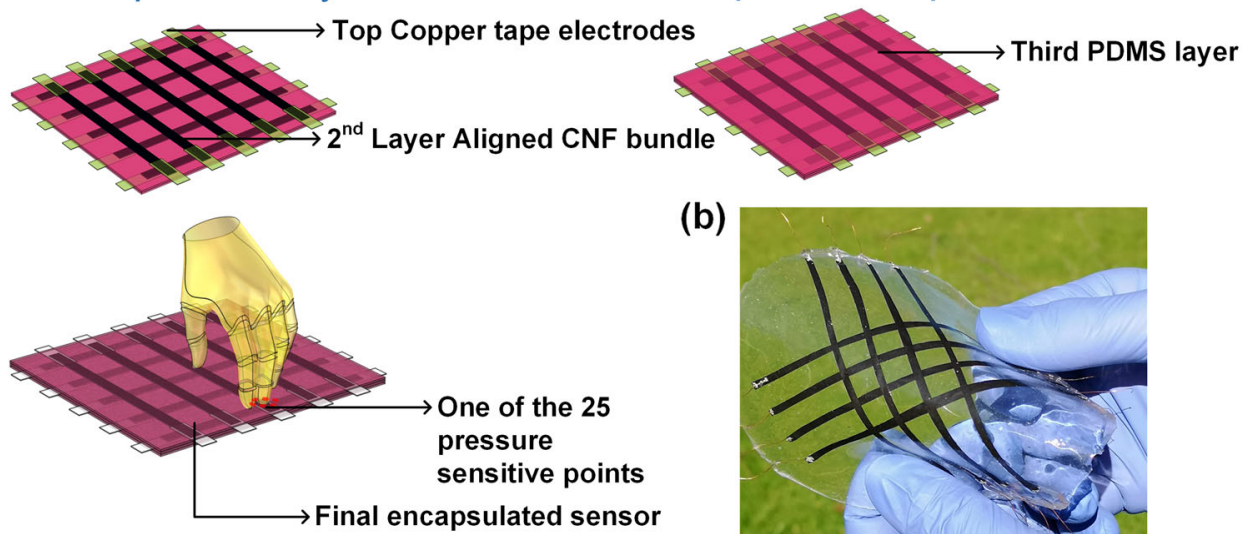

(b)

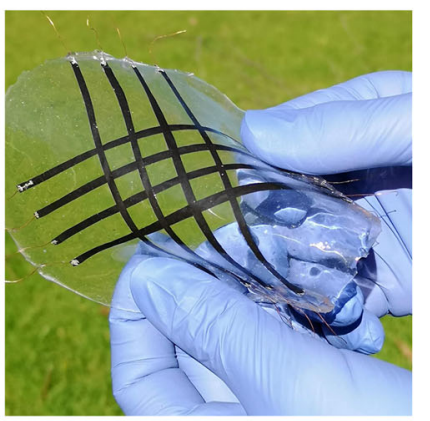

(c)

Signal profile of applied tactile test

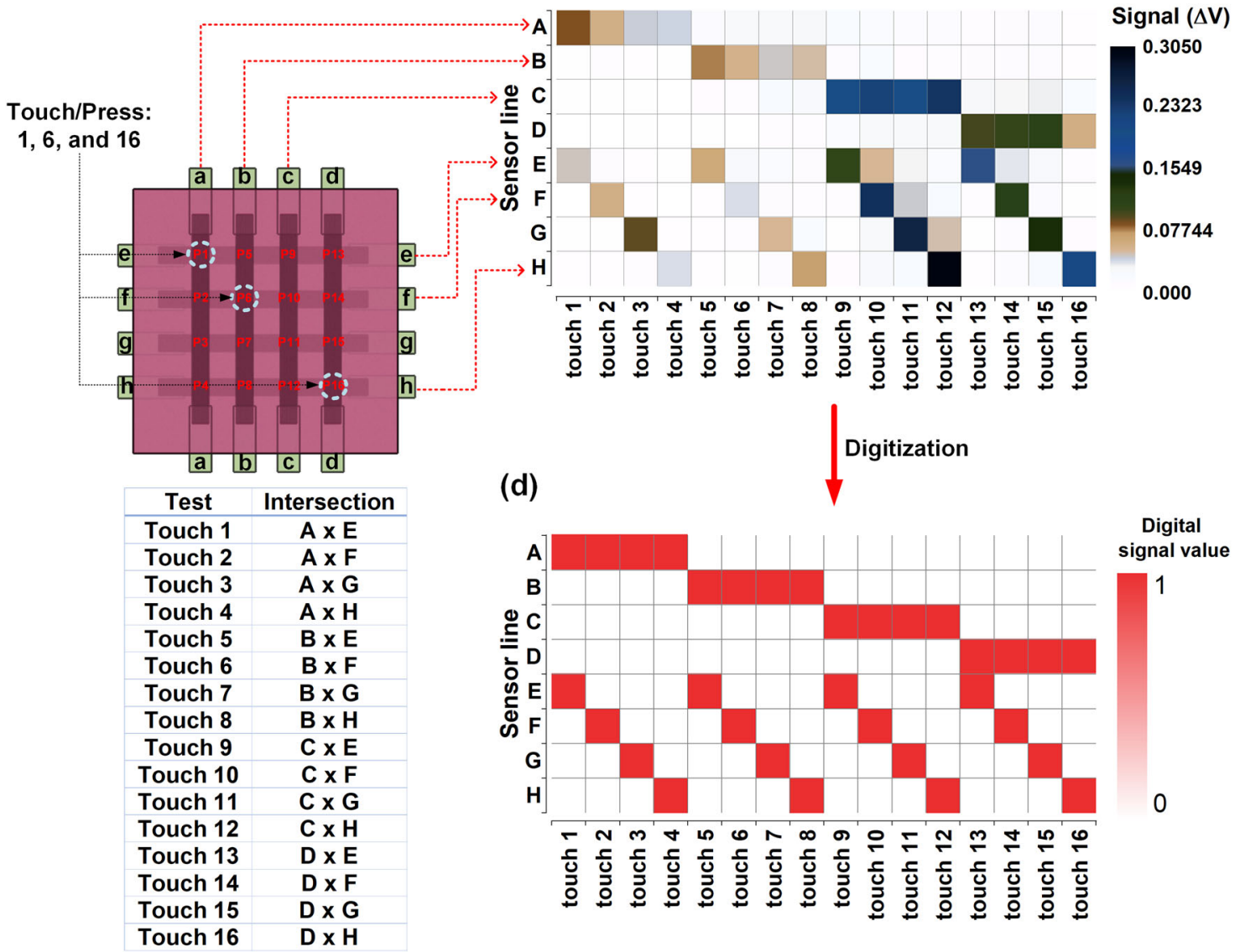

Fig. 9 Multipoint pressure/touch sensitive flexible sensor array fabrication methods. a Schematic representation of the process steps involved in fabrication of the multipoint pressure/touch sensitive flexible sensor array. b Image of the fabricated 16-point pressure/touch sensitive flexible sensor array. c Schematic diagram with the output color grid plot to show the mechanism behind the working principle of the multi-point flexible touch/pressure sensor array. $\mathbf{d}$ Color grid-plot showing the capability of the multipoint touch sensitive array to identify 16 distinct pressure sensitive points represented by unique combinations in the color-grid plot. 
(a) Glabrous skin

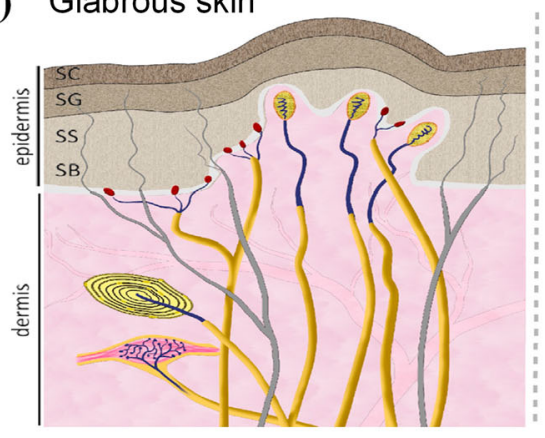

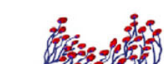

Merkel cells
(Aß SAI-LTMR)

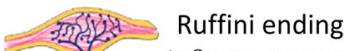

(Aß SAII-LTMR?)

Meissner corpuscle

(A $\beta$ RAI-LTMR)

Pacinian's corpuscle

(Aß RAII-LTMR)

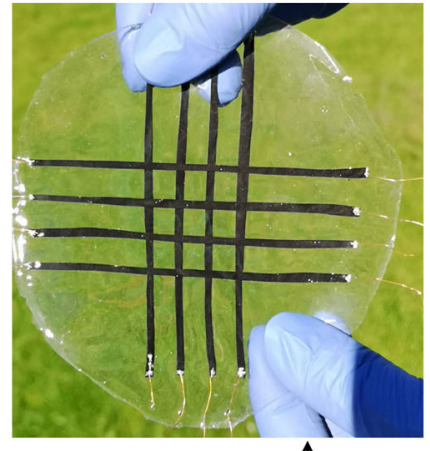

(b)
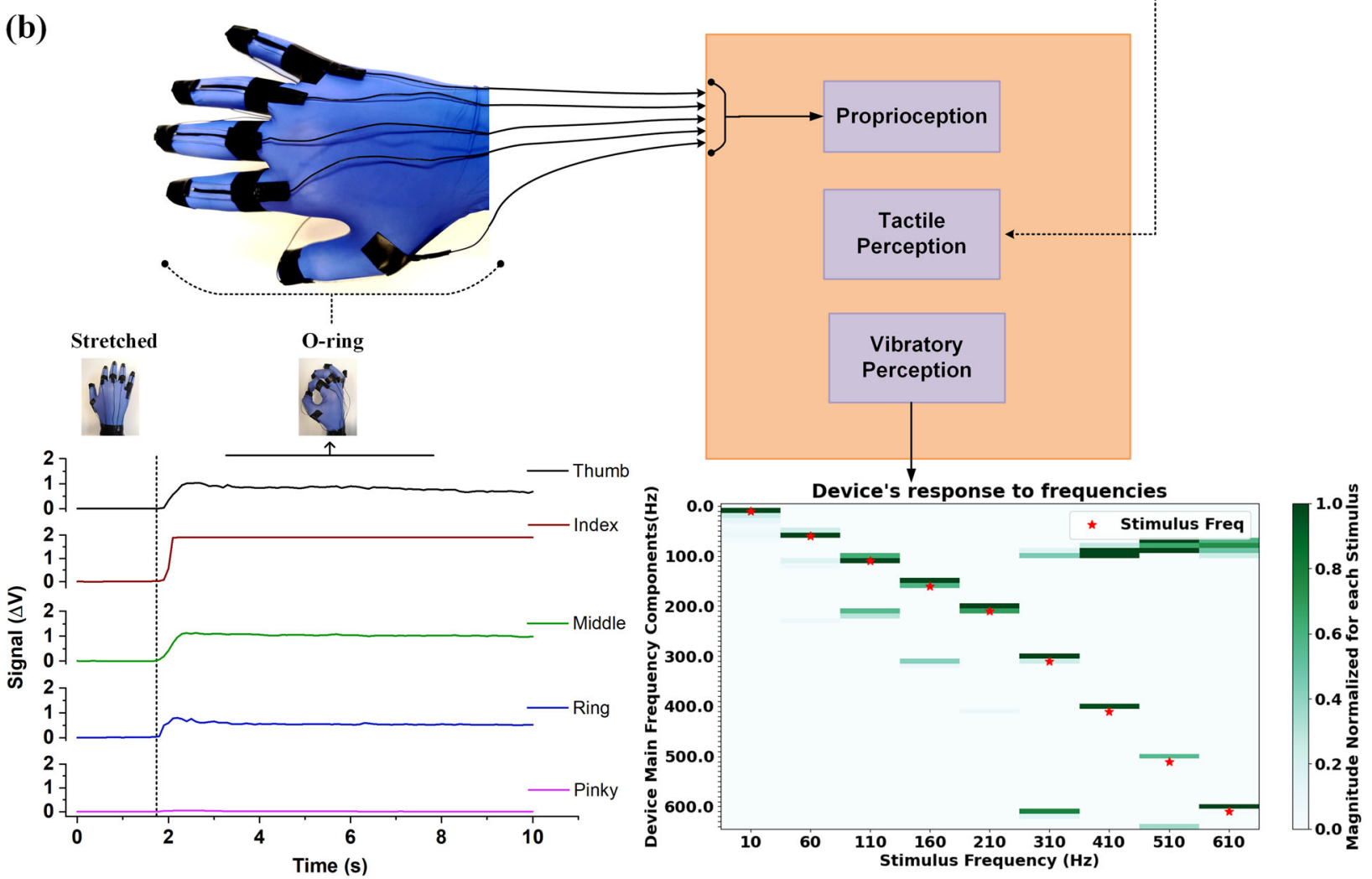

Fig. 10 Mimicking human hand employing CNF-PDMS sensors. a Schematic representation of glabrous skin with underlying RA and SALTMRs. Reproduced with permission ${ }^{37}$. 2013, Cell press. b A concept design of a neuromorphic system inspired by human hand which combines inputs from strain sensors on finger to mimic proprioception; multipoint touch detection employing tactile sensor array; and vibration detection to mimic RA-LTMRs of the glabrous skin of the human palm.

synthesized carbon nanofiber bundles. Samples having dimensions of $2 \mathrm{~cm} \times 2 \mathrm{~cm}$ were sputtered with gold and placed on an appropriate SEM stub. An acceleration voltage of $5 \mathrm{kV}$ and a spot size of 3.0 were used for conducting the imaging studies.

\section{Strain/pressure sensor fabrication}

The process steps for developing the CNF bundle strain/pressure sensor are schematically represented in Fig. 3a. A base layer of PDMS film was fabricated on which a CNF bundle bonded to copper tape electrodes on two edges (by employing conductive silver epoxy) was placed. Finally, a complete encapsulation was achieved by pouring liquid PDMS and subsequent curing. The recipe above was used for fabricating different configurations of strain and pressure sensors. For pressure sensing characterization and applications involving, gait monitoring, wrist motion-detection, and breathing monitoring, sensors comprising of $2 \times$ $2 \mathrm{~cm}$ CNF bundles were fabricated. For tensile strain sensing and finger gesture tracking applications, the sensors fabricated comprised of CNF bundles having dimensions of $4.5 \times 0.2 \mathrm{~cm}$.

\section{Sensor characterization and demonstration experiments}

For demonstrating the capability of the sensor in sensing pressure and strain stimuli, a Wheatstone bridge circuit was designed, to which the sensor was connected as an arm. The pressure sensing characterization experiments were conducted by employing an Instron 5940 UTS piezotester model with customized movable pistons. A simplified PDMS finger model having the shape of a spherical dome (with the dimensions $2 \times 1 \times$ $1 \mathrm{~cm}$ ) was attached to the top movable piston of the piezotester to simulate finger press while the experiments were conducted (schematically shown in Fig. 5a). For the development of the gesture sensing smart glove, nitrile non-powdered PPE Category III gloves were employed. For the demonstration of apparel integrability, the CNF bundle was sandwiched between two layers of the fabrics ( $99 \%$ cotton, $1 \%$ elastane) and stitching was performed with cotton thread and standard sewing needle to secure the sensing element without damaging. For all the experiments, the signal outputs from the Wheatstone bridge circuit were acquired and logged using a National Instruments data acquisition system (DAQ, NI USB-6009) with National Instruments Signal express. A constant power supply of $9 \mathrm{~V}$ was used for powering the Wheatstone bridge circuit during all the experiments. Furthermore, the output signals from the 
bridge circuit were acquired continuously at a sample acquisition rate of $2 \mathrm{kHz}$ in a differential configuration. For all the experiments, unamplified outputs of the sensors were plotted.

\section{Human motion monitoring experiments}

For demonstrating the capability of the sensor in sensing real-time human motion (gait monitoring, wrist motion-detection, and breathing monitoring), sensors comprising of $2 \times 2 \mathrm{~cm}$ CNF bundles encapsulated in PDMS were fabricated. The sensor was secured safely to the desired body parts utilizing non-toxic adhesive tapes. Subsequently, the sensor was connected to a Wheatstone bridge circuit and the voltage output signal from the bridge circuit in response to motion (entailing wrist bending, breathing, and gait) was continuously logged employing NI DAQ USB6009 acquisition setup. All the experiments involving human motion monitoring were conducted on a single human subject to maintain overall experimental consistency. To ensure the safety of the human test subject all general laboratory safety protocols mandated by University of Groningen's Health, Safety \& Sustainability (AMD) have been followed. To ensure the privacy of the human test subject, confidentiality has been ensured by removing relevant personal information.

\section{DATA AVAILABILITY}

The data that support the findings of this study are available from the corresponding author upon reasonable request.

Received: 27 May 2021; Accepted: 16 September 2021; Published online: 14 October 2021

\section{REFERENCES}

1. Wall, J. C. \& Turnbull, G. I. Gait asymmetries in residual hemiplegia. Arch. Phys. Med. Rehabil. 67, 550-553 (1986).

2. Ajovalasit, A. \& Zuccarello, B. Local reinforcement effect of a strain gauge installation on low modulus materials. J. Strain Anal. Eng. Des. 40, 643-653 (2005).

3. Barlian, A. A., Park, W.-T., Mallon, J. R., Rastegar, A. J. \& Pruitt, B. L. Review: semiconductor piezoresistance for microsystems. Proc. IEEE 97, 513-552 (2009).

4. Cao, L., Kim, T. S., Mantell, S. C. \& Polla, D. L. Simulation and fabrication of piezoresistive membrane type MEMS strain sensors. Sensors Actuators, A Phys. https://doi.org/10.1016/S0924-4247(99)00343-X (2000).

5. Da Silva, J. G., De Carvalho, A. A. \& Da Silva, D. D. A strain gauge tactile sensor for finger-mounted applications. IEEE Trans. Instrum. Meas. https://doi.org/10.1109/ 19.989890 (2002)

6. Amjadi, M., Yoon, Y. J. \& Park, I. Ultra-stretchable and skin-mountable strain sensors using carbon nanotubes-Ecoflex nanocomposites. Nanotechnology https://doi.org/10.1088/0957-4484/26/37/375501 (2015)

7. Qin, Y. et al. Lightweight, superelastic, and Mechanically flexible graphene/ polyimide nanocomposite foam for strain sensor application. ACS Nano 9, 8933-8941 (2015).

8. Yao, $\mathrm{H}$. et al. A flexible and highly pressure-sensitive graphene-polyurethane sponge based on fractured microstructure design. Adv. Mater. 25, 6692-6698 (2013).

9. Pang, Y. et al. Flexible, highly sensitive, and wearable pressure and strain sensors with graphene porous network structure. ACS Appl. Mater. Interfaces 8 26458-26462 (2016).

10. Boland, C. S. et al. Sensitive, high-strain, high-rate bodily motion sensors based on graphene-rubber composites. ACS Nano 8, 8819-8830 (2014).

11. Amjadi, M. et al. Highly stretchable and sensitive strain sensor based on silver nanowire-elastomer nanocomposite. ACS Nano 8, 5154-5163 (2014).

12. Zhang, S. et al. Ultrasensitive and highly compressible piezoresistive sensor based on polyurethane sponge coated with a cracked cellulose nanofibril/silver nanowire layer. ACS Appl. Mater. Interfaces 11, 10922-10932 (2019).

13. Kim, J. et al. Stretchable silicon nanoribbon electronics for skin prosthesis. Nat. Commun. 5, 5747 (2014).

14. Yamada, T. et al. A stretchable carbon nanotube strain sensor for human-motion detection. Nat. Nanotechnol. https://doi.org/10.1038/nnano.2011.36 (2011).

15. Kong, J.-H., Jang, N.-S., Kim, S.-H. \& Kim, J.-M. Simple and rapid micropatterning of conductive carbon composites and its application to elastic strain sensors. Carbon N. Y. 77, 199-207 (2014).

16. Liu, H. et al. Lightweight conductive graphene/thermoplastic polyurethane foams with ultrahigh compressibility for piezoresistive sensing. J. Mater. Chem. C. 5, 73-83 (2017).
17. Huang, T. et al. Porous Fibers Composed of Polymer Nanoball Decorated Graphene for Wearable and Highly Sensitive Strain Sensors. Adv. Funct. Mater https://doi.org/10.1002/adfm.201903732 (2019).

18. Liu, Q., Chen, J., Li, Y. \& Shi, G. High-performance strain sensors with fish-scale-like graphene-sensing layers for full-range detection of human motions. ACS Nano https://doi.org/10.1021/acsnano.6b03813 (2016).

19. Sengupta, D., Pei, Y. \& Kottapalli, A. G. P. Ultralightweight and 3D squeezable graphene-polydimethylsiloxane composite foams as piezoresistive sensors. ACS Appl. Mater. Interfaces 11, 35201-35211 (2019).

20. Sengupta, D. \& Prakash Kottapalli, A. G. Ultralight Weight Piezoresistive Spongy Graphene Sensors for Human Gait Monitoring Applications. 120-123, https://doi. org/10.1109/nems50311.2020.9265580 (Institute of Electrical and Electronics Engineers (IEEE), 2020).

21. Sengupta, D., Muthuram, V. \& Prakash Kottapalli, A. G. Flexible Graphene-onPDMS Sensor for Human Motion Monitoring Applications. In 2020 IEEE Sensors, 1-4, https://doi.org/10.1109/SENSORS47125.2020.9278663 (IEEE, 2020).

22. Ryu, S. et al. Extremely elastic wearable carbon nanotube fiber strain sensor for monitoring of human motion. ACS Nano 9, 5929-5936 (2015).

23. Wang, X., Qiu, Y., Cao, W. \& Hu, P. HigHly Stretchable And Conductive Core-sheath Chemical Vapor Deposition Graphene Fibers And Their Applications In Safe Strain Sensors. Chem. Mater. 27, 6969-6975 (2015).

24. Bhuyan, M. S. A., Uddin, M. N., Islam, M. M., Bipasha, F. A. \& Hossain, S. S. Synthesis of graphene. Int. Nano Lett. 6, 65-83 (2016).

25. Zhao, G. et al. The physics and chemistry of graphene-on-surfaces. Chem. Soc. Rev. 46, 4417-4449 (2017).

26. Zhang, Q., Huang, J. Q., Zhao, M. Q., Qian, W. Z. \& Wei, F. Carbon nanotube mass production: Principles and processes. ChemSusChem 4, 864-889 (2011).

27. $\mathrm{Ma}, \mathrm{Y}$. et al. A highly flexible and sensitive piezoresistive sensor based on MXene with greatly changed interlayer distances. Nat. Commun. 8, 1207 (2017).

28. Li, L. et al. Hydrophobic and Stable MXene-Polymer Pressure Sensors for Wearable Electronics. ACS Appl. Mater. Interfaces https://doi.org/10.1021/ acsami.0c00255 (2020).

29. Sharma, S., Chhetry, A., Sharifuzzaman, M., Yoon, H. \& Park, J. Y. Wearable capacitive pressure sensor based on mxene composite nanofibrous scaffolds for reliable human physiological signal acquisition. ACS Appl. Mater. Interfaces https:// doi.org/10.1021/acsami.0c05819 (2020).

30. Ren, Z. et al. Fully Elastic and Metal-Free Tactile Sensors for Detecting both Normal and Tangential Forces Based on Triboelectric Nanogenerators. Adv. Funct. Mater. https://doi.org/10.1002/adfm.201802989 (2018).

31. Tao, J. et al. Self-powered tactile sensor array systems based on the triboelectric effect. Adv. Funct. Mater. 29, 1806379 (2019).

32. Li, C. et al. Flexible dome and bump shape piezoelectric tactile sensors using PVDF-TrFE copolymer. J. Microelectromech. Syst. 17, 334-341 (2008).

33. Kim, M. S., Ahn, H. R., Lee, S., Kim, C. \& Kim, Y. J. A dome-shaped piezoelectric tactile sensor arrays fabricated by an air inflation technique. Sensors Actuators, $A$ Phys. https://doi.org/10.1016/j.sna.2014.02.023 (2014).

34. Sengupta, D. et al. Characterization of single polyvinylidene fluoride (PVDF) nanofiber for flow sensing applications. AIP Adv. 7, 105205 (2017).

35. Van Lier, G., Van Alsenoy, C., Van Doren, V. \& Geerlings, P. Ab initio study of the elastic properties of single-walled carbon nanotubes and graphene. Chem. Phys. Lett. https://doi.org/10.1016/S0009-2614(00)00764-8 (2000).

36. Rinaldi, A., Tamburrano, A., Fortunato, M. \& Sarto, M. S. A Flexible and highly sensitive pressure sensor graphene nanoplatelets. Sensors https://doi.org/ 10.3390/s16122148 (2016).

37. Abraira, V. E. \& Ginty, D. D. The sensory neurons of touch. Neuron https://doi.org/ 10.1016/j.neuron.2013.07.051 (2013).

38. Sengupta, D. et al. Single and bundled carbon nanofibers as ultralightweight and flexible piezoresistive sensors. npj Flex. Electron. 4, 9 (2020).

39. Sengupta, D., Trap, D. \& Kottapalli, A. G. P. Piezoresistive carbon nanofiber-based cilia-inspired flow sensor. Nanomaterials 10, 211 (2020).

40. Cai, J., Chawla, S. \& Naraghi, M. Piezoresistive effect of individual electrospun carbon nanofibers for strain sensing. Carbon N. Y. 77, 738-746 (2014).

41. Ruz, P. et al. Structural evolution of turbostratic carbon: Implications in H2 storage. Solid State Sci. 62, 105-111 (2016).

42. Paschen, S., Bussac, M. N., Zuppiroli, L., Minder, E. \& Hilti, B. Tunnel junctions in a polymer composite. J. Appl. Phys. 78, 3230-3237 (1995).

43. Simmons, J. G. Generalized formula for the electric tunnel effect between similar electrodes separated by a thin insulating film. J. Appl. Phys. 34, 1793-1803 (1963).

44. Yu, Y., Song, G. \& Sun, L. Determinant role of tunneling resistance in electrical conductivity of polymer composites reinforced by well dispersed carbon nanotubes. J. Appl. Phys. 108, 084319 (2010).

45. Ounaies, Z., Park, C., Wise, K. E., Siochi, E. J. \& Harrison, J. S. Electrical properties of single wall carbon nanotube reinforced polyimide composites. Compos. Sci. Technol. 63, 1637-1646 (2003). 
14

46. Xiao, X. et al. High-strain sensors based on $\mathrm{ZnO}$ nanowire/polystyrene hybridized flexible films. Adv. Mater. 45, 5440-5444 (2011).

47. Park, J. J., Hyun, W. J., Mun, S. C., Park, Y. T. \& Park, O. O. Highly stretchable and wearable graphene strain sensors with controllable sensitivity for human motion monitoring. ACS Appl. Mater. Interfaces 7, 6317-6324 (2015).

48. Asakawa, D. S., Crocker, G. H., Schmaltz, A. \& Jindrich, D. L. Fingertip forces and completion time for index finger and thumb touchscreen gestures. J. Electromyogr. Kinesiol. 34, 6-13 (2017).

\section{ACKNOWLEDGEMENTS}

This research was supported by financial support from the University of Groningen's start-up grant and Dutch Research Council (NWO) through the NWA Idea Generator project (NWA.1228.192.279) awarded to A.G.P.K. The authors would like to express their sincere gratitude to Professor Chee Yee Kwok and Dr. Aron Michael from the University of New South Wales for their help with SEM imaging. The authors acknowledge the contribution of Mr. Michele Mastella for inputs regarding vibration response test. The authors would also like to express their gratitude to Prof. Dr. Beatriz Noheda Pinuaga from Zerinke Institute for Advanced Materials (ZIAM) and Dr. Mónica Isela Acuautla Meneses from Engineering and Technology Institute Groningen (ENTEG) for providing training and access to Instron 5940 UTS.

\section{AUTHOR CONTRIBUTIONS}

D.S.G. conducted the electrospinning, carbon nanofiber synthesis, and characterization. D.S.G. and J.R. conducted the strain sensor fabrication, sensor testing, and wrote the manuscript. D.S.G. and A.G.P.K. conceptualized the idea. A.G.P.K. conceived and supervised the project. All authors contributed to the discussion of the results and to the critical reading of the manuscript.

\section{COMPETING INTERESTS}

The authors declare no competing interests.

\section{ADDITIONAL INFORMATION}

Supplementary information The online version contains supplementary material available at https://doi.org/10.1038/s41528-021-00126-8.

Correspondence and requests for materials should be addressed to Ajay Giri Prakash Kottapalli.

Reprints and permission information is available at http://www.nature.com/ reprints

Publisher's note Springer Nature remains neutral with regard to jurisdictional claims in published maps and institutional affiliations.

(c) Open Access This article is licensed under a Creative Commons adaptation, distribution and reproduction in any medium or format, as long as you give appropriate credit to the original author(s) and the source, provide a link to the Creative Commons license, and indicate if changes were made. The images or other third party material in this article are included in the article's Creative Commons license, unless indicated otherwise in a credit line to the material. If material is not included in the article's Creative Commons license and your intended use is not permitted by statutory regulation or exceeds the permitted use, you will need to obtain permission directly from the copyright holder. To view a copy of this license, visit http://creativecommons. org/licenses/by/4.0/.

(c) The Author(s) 2021 